\title{
A new method for 3-dimensional roadway design using visualization techniques
}

\author{
G. Karri \& M. K. Jha \\ Department of Civil Engineering, Morgan State University, USA
}

\begin{abstract}
Traditionally, geometric design of roadways is a two-stage process in which the horizontal alignment is designed first and then the vertical alignment is designed based on the former. A design change in horizontal alignment will affect the vertical alignment design. Also, the traditional two-stage road design may lead to critical design errors, such as a section of the road with a vertical valley curve and a sharp horizontal curve together will lead to an illusion of a lesser sharp horizontal curve when driving. This is due to the fact that the vertical curve may be overlooked as it cannot be traced by the traditional two-stage design process. One can thus see that this two-stage process is cumbersome and subject to error. Literature review shows that this error-prone and cumbersome process can be improved by visualizing roads three dimensionally in a single stage. In this paper we propose a method in which the traditional two-stage design process can be reduced to a single-stage design process by developing a three-dimensional (3D) roadway using visualization techniques. The roadway is designed using elements like 3D lines and piecewise polynomials (splines). The design involves identifying control points on a given terrain, through which the roadway passes, and finding suitable 3D lines and/or splines that connect those control points. Mathematical formulations to calculate the 3D lines and splines are presented. The paper concludes with the discussion of the application of the proposed methodology to full-scale efficient roadway design and directions for future research.
\end{abstract}

Keywords: roadway geometric design, visualization, splines, $3 D$. 


\section{Introduction}

Traditional geometric design of roadways involves the design of horizontal and vertical alignments for a smooth and safe riding of vehicles. Some of critical information for geometric design may not be available in horizontal alignment or in vertical alignment because of the planar projection of highway on to XY plane or XZ plane (Cartesian coordinate system). For example, a designer may not be able to adjudge the sight distance by looking at horizontal or vertical alignment, because in horizontal alignment the elevation information is not available and in vertical alignment the horizontal alignment of road is not available. Another problem that can be seen in 2D geometric design method is that the designer may not identify an illusion effect when horizontal curve appears simultaneously with vertical curve. Without identifying the effect if the roadway is constructed, the simultaneous appearance of horizontal and vertical curve may give an illusion to the driver that highway is safe for higher speeds but in practice it may not be. These problems to some extent can be solved by designing highways using three dimensional visualization techniques. Using these techniques the designer can view the alignment three dimensionally and identify the errors that cannot be identified either in horizontal or vertical alignment. Therefore, one can see that three dimensional geometric design of roadways has more advantages when compared to the two dimensional geometric design.

Literature review shows that not many three dimensional geometric road design methods are available. Gibreel et al. [2] described the calculation of operational speeds on roadways using three dimensional alignments. But, the method described applies to a particular type of roadway and is not a general purpose method. Jha et al. [3] presented a model for highway alignment using GIS, genetic algorithms, and computer visualization techniques. Computer visualization is basically used for showing different alternative solutions but no method is presented for 3D geometric design of highways. Kang et al. [4] described 3D alignment optimization but the method basically deals with finding an optimal alignment between two given points. The roadway is designed based on existing 2D design methods. Kuhn [5] presented a 3D geometric design method for roadways using combination of fixed, dialogue and coupling elements; but, the paper does not provide information of the choice of elements.

From the above discussion it is clear that there is a need for exploiting 3D geometric design methods and latest visualization technologies for improved roadway design.

\section{Methodology}

The methodology for 3-dimensional roadway design is based on the existing 2-dimensional horizontal and vertical design methods. In 2D design methods, the roadway alignment is composed of design elements that are a combination of straight lines, circular curves, parabolic curves, and spiral curves. In either horizontal or vertical alignment, a change in straight road (straight line) direction is incorporated through a curve, i.e., two straight portions of a road, which are 
adjacent and have different directions are connected with a curve. Circular curves are used in horizontal alignment design and parabolic curves are used in vertical alignment design, to join the straight portions. Similar approach is used for three dimensional roadway designs.

\subsection{Design elements}

In our approach the alignment is composed of two design elements, 3D straight lines and 3D spline curves. The 3D straight lines are connected with spiral curves.

\subsubsection{D straight lines}

A 3D straight line consists of a start and an end point. In Cartesian coordinate system the start point is represented as $\left(\mathrm{x}_{\mathrm{s}}, \mathrm{y}_{\mathrm{s}}, \mathrm{z}_{\mathrm{s}}\right)$ and the end point is represented as $\left(x_{e}, y_{e}, z_{e}\right)$. The location of the start and end point gives the direction of the straight line. A typical 3D straight line is given in Figure 1.

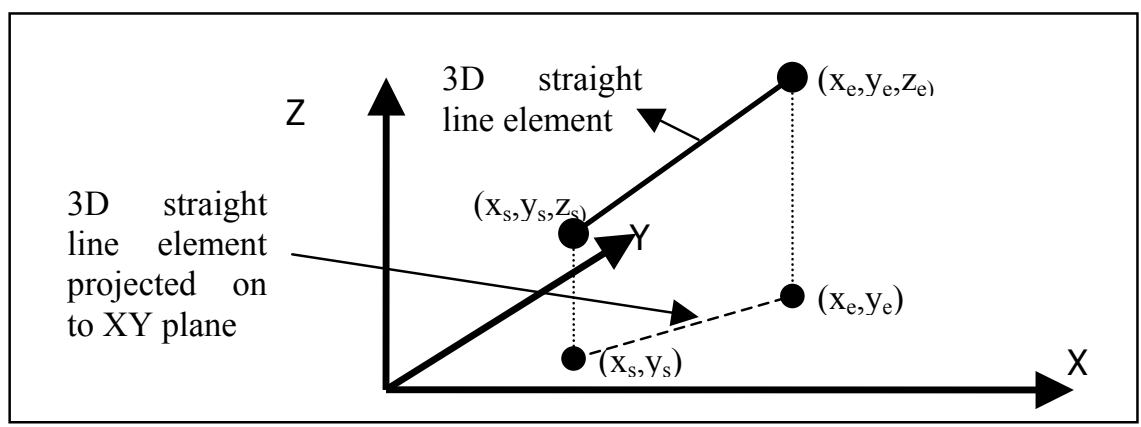

Figure 1: A typical 3D straight line.

\subsubsection{D spline curves}

Given a set of control points through which the designer wants the alignment to pass, polynomial curves can be used to pass through the control points. But, there are two disadvantages of using polynomial curves.

1. There will be no control on the adjustment of the curve between two control points for design purposes.

2. As the number of control points increases, the order of the polynomial increases and hence number of coefficients of polynomial also increases.

Instead of using one polynomial to pass through control points, one can use piecewise polynomials to reduce above said problems. These piecewise polynomials together are termed as spline curves. Splines form a good fit for given control points and intermediate points can be interpolated easily [6. 7]. In the proposed design approach, cubic splines (of order 4) are used. 
Let $C=\{0,1,2, . . l-1\}$ denote $l$ knots (which are control points in this methodology and a part of the total set of control points). These knots are in a sequence. Let $c \in C$. $c=0$ denotes the first knot in the sequence and $c=l-1$ denotes the $l^{\text {th }}$ knot. Let $\left(x_{c}, y_{c}, z_{c}\right)$ be the Cartesian coordinates of a knot in $C$. Let $X=\left\{x_{0}, x_{1}, \ldots x_{l-1}\right\}$ be the set of $x$ coordinates of all the $l$ knots. Let $Y=\left\{y_{0}, y_{1}, . . y_{l-1}\right\}$ be the set of $y$ coordinates of all the $l$ knots. Let $Z=$ $\left\{z_{0}, z_{1}, . . z_{l-1}\right\}$ be the set of $z$ coordinates of all the $l$ knots. As explained earlier, we connect these $l$ knots with a spline. The spline will have $l-1$ piecewise polynomials.

The parametric functions of $p^{\text {th }}$ piecewise polynomial of a spline that is between knots $c$ and $c+1$ is given below.

$$
\begin{aligned}
& x_{t}^{p}=a_{p x} t^{3}+b_{p x} t^{2}+c_{p x} t+d_{p x} \\
& y_{t}^{p}=a_{p y} t^{3}+b_{p y} t^{2}+c_{p y} t+d_{p y} \\
& z_{t}^{p}=a_{p z} t^{3}+b_{p z} t^{2}+c_{p z} t+d_{p z}
\end{aligned}
$$

where

$$
t \in[c, c+1)
$$

$p=c+1 . \quad p$ denotes the $p^{\text {th }}$ polynomial piece of the spline that is between knots $c$ and $c+1$. For example $p=2$ denotes the second polynomial of the spline that is between the knots 1 and 2 .

$x_{t}^{p}=p^{\text {th }}$ piece $x$ coordinate considered at position $\mathrm{t}$.

$y_{t}^{p}=p^{\text {th }}$ piece $y$ coordinate considered at position $\mathrm{t}$.

$z_{t}^{p}=p^{\text {th }}$ piece $z$ coordinate considered at position $\mathrm{t}$.

$a_{p x}, b_{p x}, c_{p x}, d_{p x}$ are coefficients of the $p^{t h}$ polynomial piece in $X$

$a_{p y}, b_{p y}, c_{p y}, d_{p y}$ are coefficients of the $p^{\text {th }}$ polynomial piece in $Y$

$a_{p z}, b_{p z}, c_{p z}, d_{p z}$ are coefficients of the $p^{\text {th }}$ polynomial piece in $Z$

Many algorithms are available which can be used to get the coefficients of piecewise polynomials. Examples include cardinal splines, Catmull Rom splines, 
and Kochanek Bartels Splines [1, 6, 7]. A typical 3D spline is shown in Figure 2. For more details on splines, the readers may refer to any computer graphics book on using splines for graphics.

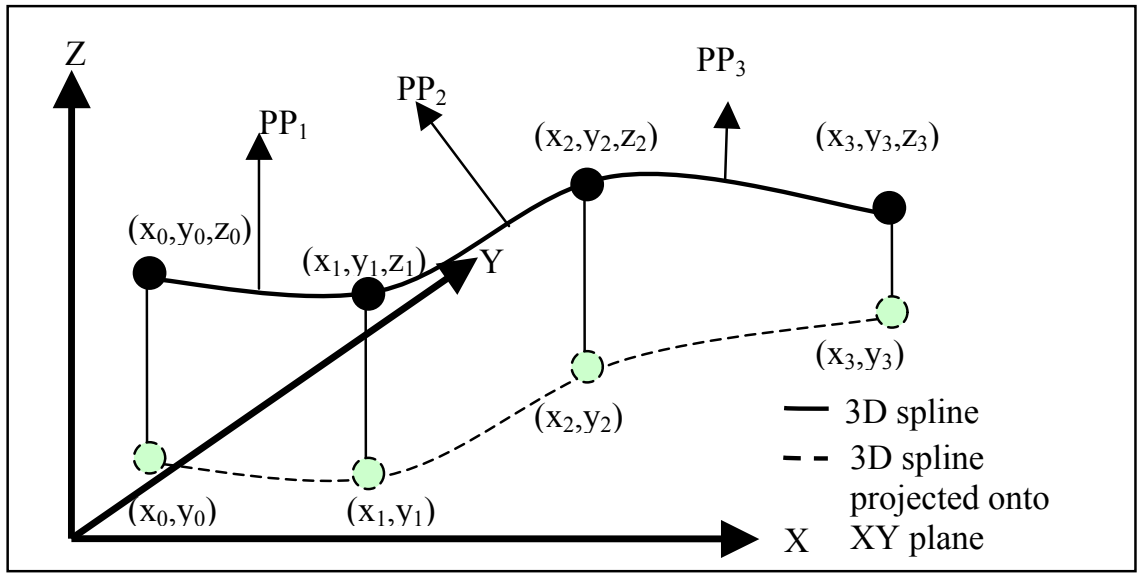

Figure 2: A typical 3D spline. Here 3D spline has three piecewise polynomials $\mathrm{PP}_{1}, \mathrm{PP}_{2}, \mathrm{PP}_{3}$ with four knots.

\subsection{Design procedure}

Given a 3D terrain, the designer has to select some control points over the terrain through which the alignment should pass. Keeping these control points in view, the designer best fits 3D straight lines based on the control points' positions. The number of 3D straight line design elements depends on the site conditions. Then the designer fits a spline element that passes through control points that are between two consecutive straight line elements. The designer can change the shape of the spline by adding additional control points or by changing the position of the existing control points. Then the designer needs to check the alignment for technical and safety issues.

\section{An example}

\subsection{An alignment created using existing methods}

A roadway alignment is created using existing methods for experimental purpose. The alignment created has three design elements namely straight-lines, clothoids, circular curves in horizontal alignment and straight-lines, and parabolic curves in vertical alignment. The geometric details are given in Tables 1 and 2 and Figure 3. 
Table 1: Geometric details for horizontal alignment.

\begin{tabular}{|l|c|c|c|c|c|c|}
\hline \multirow{2}{*}{ Type } & \multicolumn{2}{|c|}{ Start } & \multicolumn{2}{c|}{ End } & Length & \multirow{2}{*}{ Radius(m) } \\
\cline { 2 - 7 } & $\mathbf{x}$ & $\mathbf{y}$ & $\mathbf{X}$ & $\mathbf{y}$ & $\mathbf{m}$ & \\
\hline Linear & 17612.47 & 22314.02 & 17873.51 & 22102.24 & 336.14 & \\
\hline Clothoid & 17873.51 & 22102.24 & 17905.12 & 22077.74 & 40 & \\
\hline Circular & 17905.12 & 22077.74 & 18118.9 & 22025.55 & 225.32 & -300 \\
\hline Clothoid & 18118.9 & 22025.55 & 18158.24 & 22032.73 & 40 & \\
\hline Linear & 18158.24 & 22032.73 & 18355.63 & 22073.32 & 201.51 & \\
\hline Clothoid & 18355.63 & 22073.32 & 18394.97 & 22080.5 & 40 & \\
\hline Circular & 18394.97 & 22080.5 & 18735.36 & 21769.4 & 525.95 & 300 \\
\hline Clothoid & 18735.36 & 21769.4 & 18731.73 & 21729.58 & 40 & \\
\hline Linear & 18731.73 & 21729.58 & 18725.27 & 21672.58 & 57.36 & \\
\hline Clothoid & 18725.27 & 21672.58 & 18723.42 & 21632.69 & 40 & \\
\hline Circular & 18723.42 & 21632.69 & 18855.57 & 21546.83 & 181.5 & -100 \\
\hline Clothoid & 18855.57 & 21546.83 & 18891.26 & 21564.72 & 40 & \\
\hline Linear & 18891.26 & 21564.72 & 19163.05 & 21724.43 & 315.23 & \\
\hline Clothoid & 19163.05 & 21724.43 & 19197.97 & 21743.92 & 40 & \\
\hline Circular & 19197.97 & 21743.92 & 19557.23 & 21674.33 & 393.56 & 300 \\
\hline Clothoid & 19557.23 & 21674.33 & 19582.35 & 21643.21 & 40 & \\
\hline Linear & 19582.35 & 21643.21 & 19875.77 & 21262.69 & 480.51 & \\
\hline
\end{tabular}

Table 2: $\quad$ Geometric details for vertical alignment.

\begin{tabular}{|l|c|c|c|c|c|c|c|}
\hline \multirow{2}{*}{ Type } & \multicolumn{3}{|c|}{ Start } & \multicolumn{3}{c|}{ End } & $\begin{array}{c}\text { Length } \\
(\mathbf{m})\end{array}$ \\
\cline { 2 - 8 } & Station & Elevation & Grade & Station & Elevation & Grade & 20.68 \\
\hline Linear & $0+41.78$ & 371.96 & $-20.42 \%$ & $0+62.46$ & 367.74 & $-20.42 \%$ & 2.67 \\
\hline Parabola & $0+62.46$ & 367.74 & $-20.42 \%$ & $5+12.46$ & 413.61 & $40.80 \%$ & 450 \\
\hline Linear & $5+12.46$ & 413.61 & $40.80 \%$ & $5+71.66$ & 437.77 & $40.80 \%$ & 59.2 \\
\hline Parabola & $5+71.66$ & 437.77 & $40.80 \%$ & $9+71.66$ & 406.85 & $-56.26 \%$ & 400 \\
\hline Linear & $9+71.66$ & 406.85 & $-56.26 \%$ & $11+52.94$ & 304.85 & $-56.26 \%$ & 181.28 \\
\hline Parabola & $11+52.94$ & 304.85 & $-56.26 \%$ & $14+52.94$ & 262.48 & $28.01 \%$ & 300 \\
\hline Linear & $14+52.94$ & 262.48 & $28.01 \%$ & $15+17.21$ & 280.48 & $28.01 \%$ & 64.27 \\
\hline Parabola & $15+17.21$ & 280.48 & $28.01 \%$ & $17+17.21$ & 273.98 & $-34.52 \%$ & 200 \\
\hline Linear & $17+17.21$ & 273.98 & $-34.52 \%$ & $20+50.22$ & 159.04 & $-34.52 \%$ & 333.01 \\
\hline Linear & $20+50.22$ & 159.04 & $-37.44 \%$ & $22+91.26$ & 68.81 & $-37.44 \%$ & 241.04 \\
\hline Parabola & $22+91.26$ & 68.81 & $-37.44 \%$ & $25+91.26$ & 5.05 & $-5.07 \%$ & 300 \\
\hline Linear & $25+91.26$ & 5.05 & $-5.07 \%$ & $27+23.80$ & -1.68 & $-5.07 \%$ & 132.53 \\
\hline
\end{tabular}




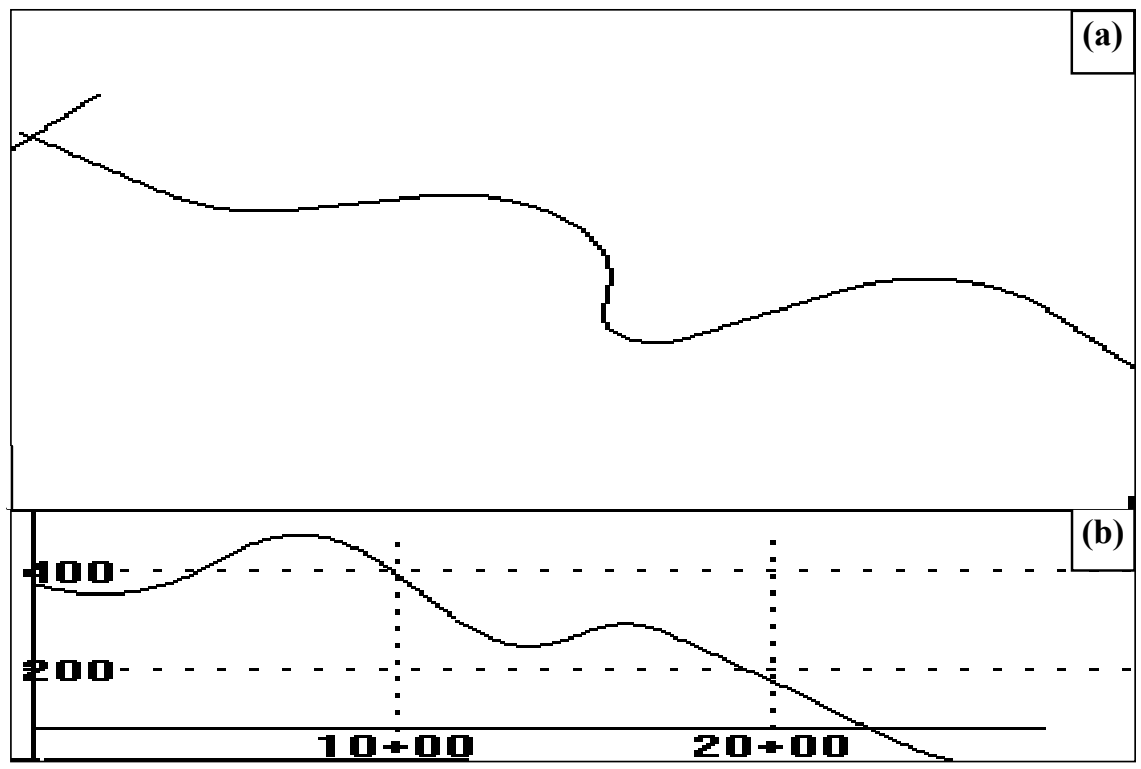

Figure 3: (a) Horizontal alignment for geometric details shown in Table 1. (b) Vertical alignment for geometric details shown in Table 2.

\subsection{Alignment created using the proposed method}

A total of 14 control points are used to design the alignment.

Table 3: $\quad$ Geometric details for a 3D alignment.

\begin{tabular}{|c|c|l|c|c|c|c|c|c|}
\hline \multirow{2}{*}{$\begin{array}{c}\text { Control } \\
\text { Point }\end{array}$} & Control & \multirow{2}{*}{ Point } & Type & \multicolumn{3}{|c|}{ Start } & \multicolumn{3}{c|}{ End } \\
\cline { 5 - 9 } & & $\mathrm{X}$ & $\mathrm{Y}$ & $\mathrm{Z}$ & $\mathrm{X}$ & $\mathrm{y}$ & $\mathrm{Z}$ \\
\hline 1 & 2 & Linear & 18012 & 20161 & 371.81 & 18136 & 20061 & 352.35 \\
\hline 2 & 3 & Spline & 18136 & 20061 & 352.35 & 18407 & 19899 & 417.26 \\
\hline 3 & 4 & Linear & 18407 & 19899 & 417.26 & 18577 & 19909 & 464.35 \\
\hline 4 & 5 & Spline & 18577 & 19909 & 464.35 & 18692 & 19941 & 465.63 \\
\hline 5 & 6 & Linear & 18692 & 19941 & 465.63 & 18811 & 19957 & 427.20 \\
\hline 6 & 7 & Spline & 18811 & 19957 & 427.2 & 18945 & 19921 & 350.36 \\
\hline 7 & 8 & Linear & 18945 & 19921 & 350.36 & 19043 & 19837 & 280.57 \\
\hline 8 & 9 & Spline & 19043 & 19837 & 280.57 & 19103 & 19650 & 251.67 \\
\hline 9 & 10 & Linear & 19103 & 19650 & 251.67 & 19091 & 19531 & 281.58 \\
\hline 10 & 11 & Spline & 19091 & 19531 & 281.58 & 19245 & 19431 & 261.89 \\
\hline 11 & 12 & Linear & 19245 & 19431 & 261.89 & 19547 & 19608 & 139.59 \\
\hline 12 & 13 & Spline & 19547 & 19608 & 139.59 & 19928 & 19544 & 11.022 \\
\hline 13 & 14 & Linear & 19928 & 19544 & 11.02 & 20051 & 19386 & -1.73 \\
\hline
\end{tabular}


Table 4: $\quad$ Spline elements' geometric properties.

\begin{tabular}{|c|c|c|c|c|c|c|c|c|}
\hline \multirow{3}{*}{$\begin{array}{c}\text { Start } \\
\text { Control } \\
\text { Point }\end{array}$} & \multirow{3}{*}{$\begin{array}{c}\text { End } \\
\text { Control } \\
\text { Point }\end{array}$} & \multirow{2}{*}{\multicolumn{2}{|c|}{ c }} & \multirow{3}{*}{$\begin{array}{c}\text { Comp- } \\
\text { onent }\end{array}$} & \multicolumn{4}{|c|}{$x_{t}^{p}=a t^{3}+b t^{2}+c t+d$} \\
\hline & & & & & \multicolumn{4}{|c|}{ Coefficients } \\
\hline & & $\begin{array}{c}\text { Start } \\
\text { Knot } \\
\text { Value } \\
\end{array}$ & $\begin{array}{c}\text { End } \\
\text { Knot } \\
\text { Value } \\
\end{array}$ & & $\mathbf{a}$ & b & c & d \\
\hline \multirow{3}{*}{2} & \multirow{3}{*}{3} & \multirow{3}{*}{0} & \multirow{3}{*}{1} & $x_{t}^{1}$ & -245.99 & 392.03 & 124.25 & 18136 \\
\hline & & & & $y_{t}^{1}$ & 232.75 & -293.54 & -100.81 & 20061 \\
\hline & & & & $z_{t}^{1}$ & -102.2 & 186.58 & -19.464 & 352.35 \\
\hline \multirow{3}{*}{4} & \multirow{3}{*}{5} & \multirow{3}{*}{0} & \multirow{3}{*}{1} & $x_{t}^{1}$ & 58.324 & -113.36 & 170.33 & 18577 \\
\hline & & & & $y_{t}^{1}$ & -36.31 & 57.36 & 10.35 & 19909 \\
\hline & & & & $z_{t}^{1}$ & 6.0956 & -51.90 & 47.089 & 464.35 \\
\hline \multirow{3}{*}{6} & \multirow{3}{*}{7} & \multirow{3}{*}{0} & \multirow{3}{*}{1} & $x_{t}^{1}$ & -51.746 & 67.24 & 118.58 & 18811 \\
\hline & & & & $y_{t}^{1}$ & 3.3715 & -55.16 & 16.133 & 19957 \\
\hline & & & & $z_{t}^{1}$ & 45.454 & -83.86 & -38.431 & 427.2 \\
\hline \multirow{3}{*}{8} & \multirow{3}{*}{9} & \multirow{3}{*}{0} & \multirow{3}{*}{1} & $x_{t}^{1}$ & -34.206 & -3.57 & 97.825 & 19043 \\
\hline & & & & $y_{t}^{1}$ & 170.37 & -273.21 & -84.068 & 19837 \\
\hline & & & & $z_{t}^{1}$ & 17.92 & 22.97 & -69.79 & 280.57 \\
\hline \multirow{3}{*}{10} & \multirow{3}{*}{11} & \multirow{3}{*}{0} & \multirow{3}{*}{1} & $x_{t}^{1}$ & -17.441 & 183.07 & -11.923 & 19091 \\
\hline & & & & $y_{t}^{1}$ & 258.53 & -239.57 & -119.38 & 19531 \\
\hline & & & & $z_{t}^{1}$ & -53.037 & 3.45 & 29.906 & 281.58 \\
\hline \multirow{3}{*}{12} & \multirow{3}{*}{13} & \multirow{3}{*}{0} & \multirow{3}{*}{1} & $x_{t}^{1}$ & -338.27 & 417.76 & 301.89 & 19547 \\
\hline & & & & $y_{t}^{1}$ & 145.98 & -386.52 & 177.09 & 19608 \\
\hline & & & & $z_{t}^{1}$ & 122.07 & -128.33 & -122.31 & 139.59 \\
\hline
\end{tabular}

From Figure 4, one can see that the alignment sections with straight line elements obtained by the proposed method are matching with some of the existing alignment sections. The other sections, i.e., the spline curved portions of the alignment sections obtained by the proposed method are not matching with the existing curved portion of the alignment. The curved portions are not matching because only two control points are used to fit a spline. If one uses more than two control points, then the curves will match. 


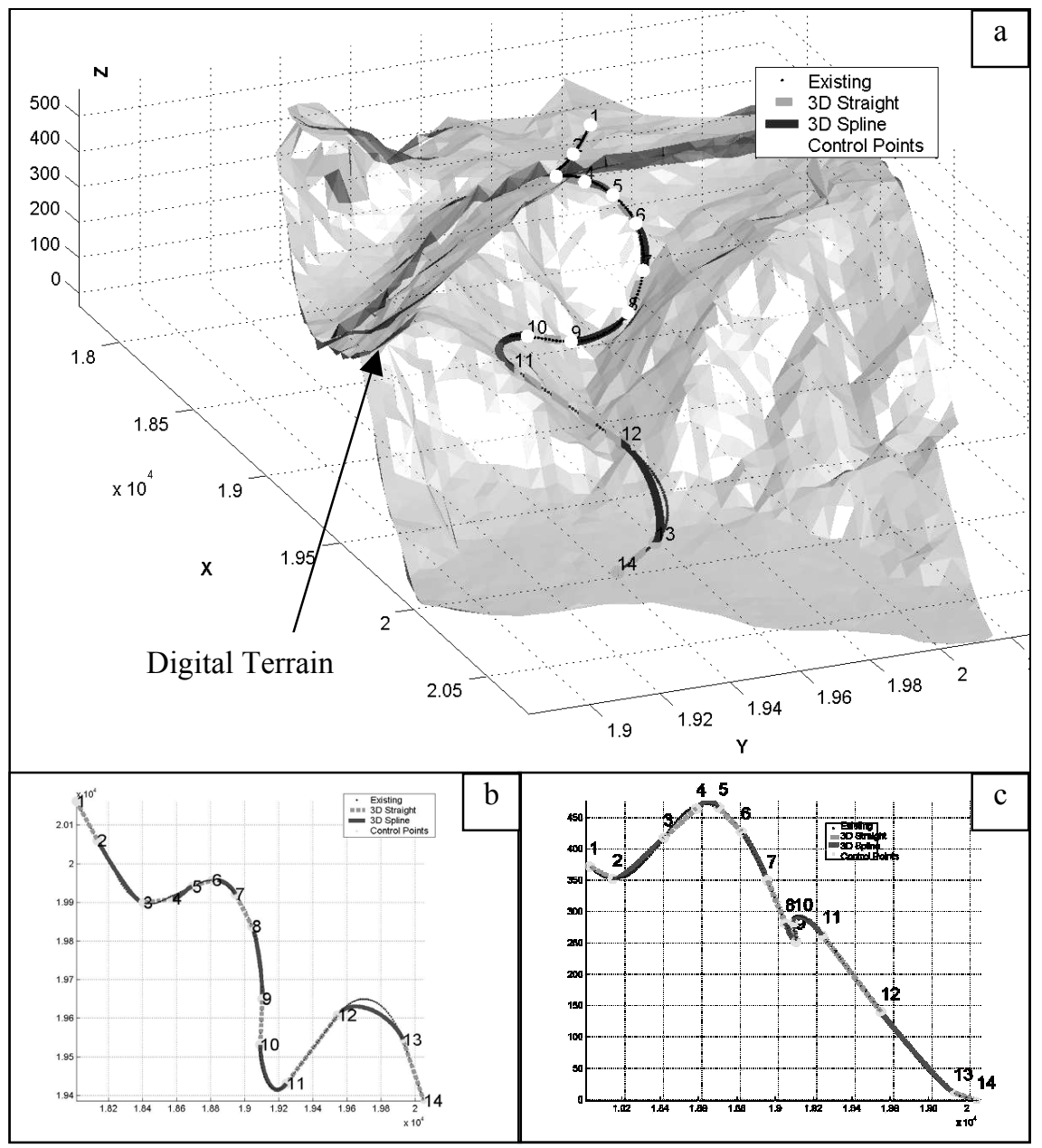

Figure 4: (a) 3D view of alignments obtained using existing and proposed methods. (b) Top view of the alignments. (c) Front view of the alignments.

\section{Discussion}

One main advantage of the proposed method over existing design methods is that the designer has more control on geometry of the highway. The designer can identify any possible errors in the 3D alignment and change the geometry accordingly by either adding more control points or changing the position of the existing control points. In the proposed method the designer can directly see the gradient and horizontal alignment of a particular roadway section simultaneously that he/she is designing, which is not possible in a $2 \mathrm{D}$ method. 
In the proposed method, we used $4^{\text {th }}$ order cubic spline. It's not necessary that one has to use cubic splines. Other types of splines are also available. The choice of the splines depends on the requirement. Different splines behave differently depending on the method used [7].

Though the methodology described helps in $3 \mathrm{D}$ design of roadways, the designed roadway needs to be checked for geometry consistency. The method does not check the alignment for comfort issues when a driver is moving on the designed roadway. Another issue related to the method is the fixing the number and position of the control points. These issues need to be considered for the improvement of the proposed method in the future works.

The control points, 3D straight lines, and the alignment using existing methods are created in Microstation. The entire code to generate 3D spline elements is developed with the Matlab software. The 3D splines are created using the control points and tangent information of 3D straight lines that are being connected with 3D splines. The code developed works well for any given set of control points and 3D straight lines. The research is carried out at the Centre for Advanced Transportation and Infrastructure Engineering Research (CATIER: www.eng.morgan.edu/ catier) at the Morgan State University.

\section{Conclusions}

The paper discusses the problems in the existing 2D methods of roadway design and the need for a single stage 3D design of roadways. A suitable 3D geometric design method that uses straight line and spline curves, is proposed for the design of roadways. The method can be further improved in the future to accommodate driver safety.

\section{References}

[1] Birkhoff, \& Boor, D., Piecewise polynomial interpolation and approximation, in: H. L. Garabedian (ed.), Proc. General Motors Symposium of 1964, Elsevier, New York and Amsterdam, pp. 164-190, 1965.

[2] Gibreel, G.M., Easa, S.M. \& El-Dimeery, I.A., Prediction of operating speed on three-dimensional highway alignments, Journal of Transportation Engineering, 2, pp.21-30, 2001.

[3] Jha, M.K., McCall, C. \& Schonfeld P., Using GIS, genetic algorithms and visualization in Highway development, Computer Aided Civil and Infrastructure Engineering, 16(6), pp.399-414, 2005.

[4] Kang, M.K., Jha, M.K. \& Schonfeld, P., 3D alignment optimization for Brookeville bypass, TRB Annual Meeting, Washington DC, CD-ROM, 2005

[5] Kuhn, W., The basics of a three-dimensional geometric design methodology, $3^{\text {rd }}$ International Symposium on Highway Geometric Design, Chicago, CD-ROM, 2005.

[6] Matlab, http://www.mathworks.com/access/helpdesk/help/toolbox/splines/

[7] Wikipedia, http://en.wikipedia.org/wiki/Spline_(mathematics) 